\title{
Exotic alleles contribute to heat tolerance in wheat under field conditions
}

Gemma Molero $^{1,2} \dagger$, Benedict Coombes ${ }^{3} \dagger$, Ryan Joynson ${ }^{3,4}$, Francisco Pinto ${ }^{1}$, Francisco J. Piñera-

Chávez ${ }^{1}$, Carolina Rivera-Amado ${ }^{1}$, Anthony Hall ${ }^{3 *}$ and Matthew P Reynolds ${ }^{1 *}$

$\dagger$ These authors contributed equally

${ }^{*}$ Corresponding authors

${ }^{1}$ International Maize and Wheat Improvement Center (CIMMYT), Texcoco 56237, Mexico

2 KWS Momont Recherche, 59246 Mons-en-Pévèle, Hauts-de-France, France (Gemma Molero's current affiliation)

${ }^{3}$ The Earlham Institute, Norwich, NR4 7UZ, UK

${ }^{4}$ Limagrain Europe, Clermont-Ferrand, France (Ryan Joynson's current affiliation)

\section{Abstract}

Global warming is one of the most significant threats to food security. With temperatures predicted to rise and extreme weather events becoming more common we must safeguard food production by developing crop varieties that are more tolerant to heat stress without compromising yield under favourable conditions. By evaluating 149 spring wheat lines in the field under yield potential and heat stressed conditions, we demonstrate how strategic integration of exotic material significantly increases yield under heat stress compared to elite lines, with no significant yield penalty under favourable conditions. Genomewide association analysis revealed three marker trait associations, which together increase yield under heat stress by over $50 \%$ compared to lines without the advantageous alleles and was associated with approximately $2^{\circ} \mathrm{C}$ lower canopy temperature. We identified an Aegilops tauschii introgression underlying the most significant of these associations. By comparing overlapping recombination of this introgressed segment between lines, we identified a $1.49 \mathrm{Mbp}$ region of the introgression responsible for this association that increases yield under heat stress by $32.4 \%$. The genes within this region were extracted from diverse Ae. tauschii genomes, revealing a novel Ae. tauschii MAPK gene, a SOC1 orthologue and a pair of type-B two-component response regulators. Incorporating these exotic alleles into breeding programmes could serve as a pre-emptive strategy to produce high yielding wheat cultivars that are resilient to the effects of future climate uncertainty with no yield penalty under favourable conditions. 


\section{Main text}

\section{Introduction}

Wheat is the most cultivated crop in the world with more than 216 million hectares grown annually (1), most of which is produced under temperate conditions (2). Heat stress is one of the major abiotic stressors that impacts global wheat production, reducing leaf area, crop duration and the efficiency of photosynthesis and respiration (3) as well as reducing floret fertility and individual grain weight (4). Together, these physiological consequences negatively impact productivity (3) with potential devastating effects. For example, in 2010 Russia saw a 30\% reduction in wheat yield during their hottest summer in 130 years (5). Cases like this could become commonplace as global warming causes temperatures to rise and extreme weather events to become more frequent. Simulations predict that yields globally will fall by on average $6 \%$ for each $1^{\circ} \mathrm{C}$ increase in temperature (6), with some regions reaching $9.1 \% \pm 5.4 \%$ per $1^{\circ} \mathrm{C}$ rise (7). Adaptation to future climate scenarios is vital to ensure global food security (8). Climatic instability, combined with environmental constraints, such as restricted supplies of irrigation water and arable land loss, emphasises the need for breeding strategies that deliver both increased yield potential during favourable cycles and resilience to abiotic stress and environmental constraints.

Such adaptation relies on genetic variation underlying the traits of interest; however, modern elite wheat material typically has limited genetic variation, particularly in the $D$ genome (9), due to historic genetic bottlenecks $(10,11)$ compounded by intensive artificial selection by breeders (12). A strategy employed by CIMMYT to increase the genetic diversity of wheat pre-breeding material is to incorporate exotic parents in their germplasm via strategic crosses $(11,13)$. The most common exotic parents used are Mexican landraces (14) and primary synthetics, which are produced by hybridising tetraploid durum wheat with Aegilops tauschii, the ancestral donor of the $D$ genome, to recreate hexaploid bread wheat (15); these synthetic lines act as a bridge to introduce durum and Ae. tauschii variation into modern hexaploid wheat. This approach has been successful in introducing disease resistance as well as drought and heat adaptive traits $(16,17)$. Landrace and synthetic material have been identified with superior biomass in comparison to elite lines under drought and heat conditions $(18,19)$ and elite lines that include landrace or synthetic material in their background have been developed in recent years for drought, heat, and yield potential conditions (20-22).

Challenges remain for the effective deployment of landrace and synthetic material. Only a small fraction of these vast collections of crop genetic resources have been evaluated for climate resilience traits and potential tradeoffs under favorable conditions have not been assessed. Currently, most of these genetic resources are unused (23) as breeders tend to avoid exotic materials because of large regions of poor recombination and a fear of linkage drag (24). Furthermore, despite evidence of the contribution of exotic material in wheat improvement, the physiological and genetic bases of heat tolerance in this material remain unclear. 
Here, we evaluate a spring wheat panel in the field containing contrasting material controlled for phenology and plant height under heat stress and yield potential conditions. We explore yield and related physiological traits and compare exotic-derived lines with elite lines. We conduct a genome-wide association study to reveal genetic associations with heat tolerance traits and evaluate their impact under favorable conditions. Finally, we identify introgressed material overlapping a marker trait association (MTA) and employ a novel method downstream of GWAS, using in silico mapping to narrow down the interval, explore recombination and extract candidate genes from introgressed genomes.

\section{Results}

\section{Physiological evaluation of HiBAP I under heat stress}

To estimate the contribution of exotic material to heat tolerance and identify its genetic bases, we evaluated the High Biomass Association Panel I (HiBAP I) for two consecutive years under yield potential and heat stressed irrigated conditions in NW-Mexico (Table S1). HiBAP I represents an unprecedented resource of genetic diversity (25). It contains 149 lines, some of which are elite while others contain exotic material from Mexican landraces, synthetics, and wild relatives (Fig 1a, Table S2). All lines have agronomically acceptable backgrounds and a restricted range of phenology and plant height (21) which allows traits of interest to be evaluated without confounding effects. 
a

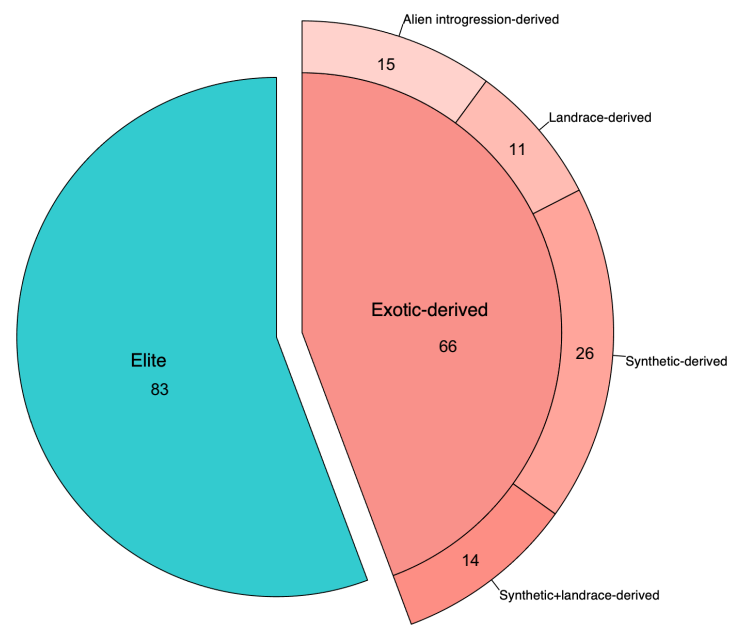

b

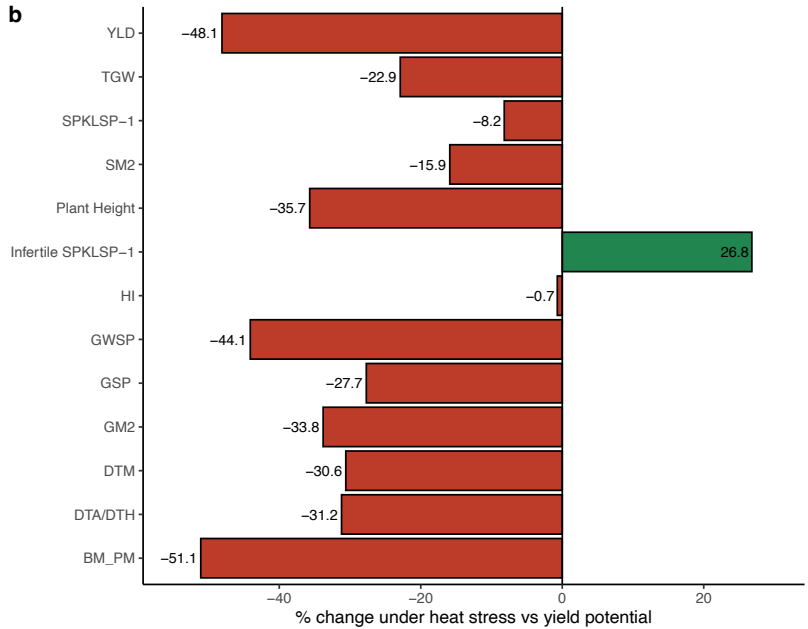

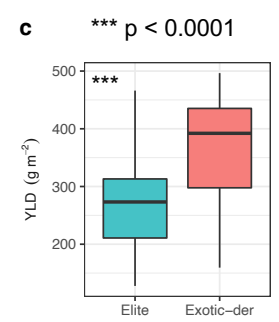
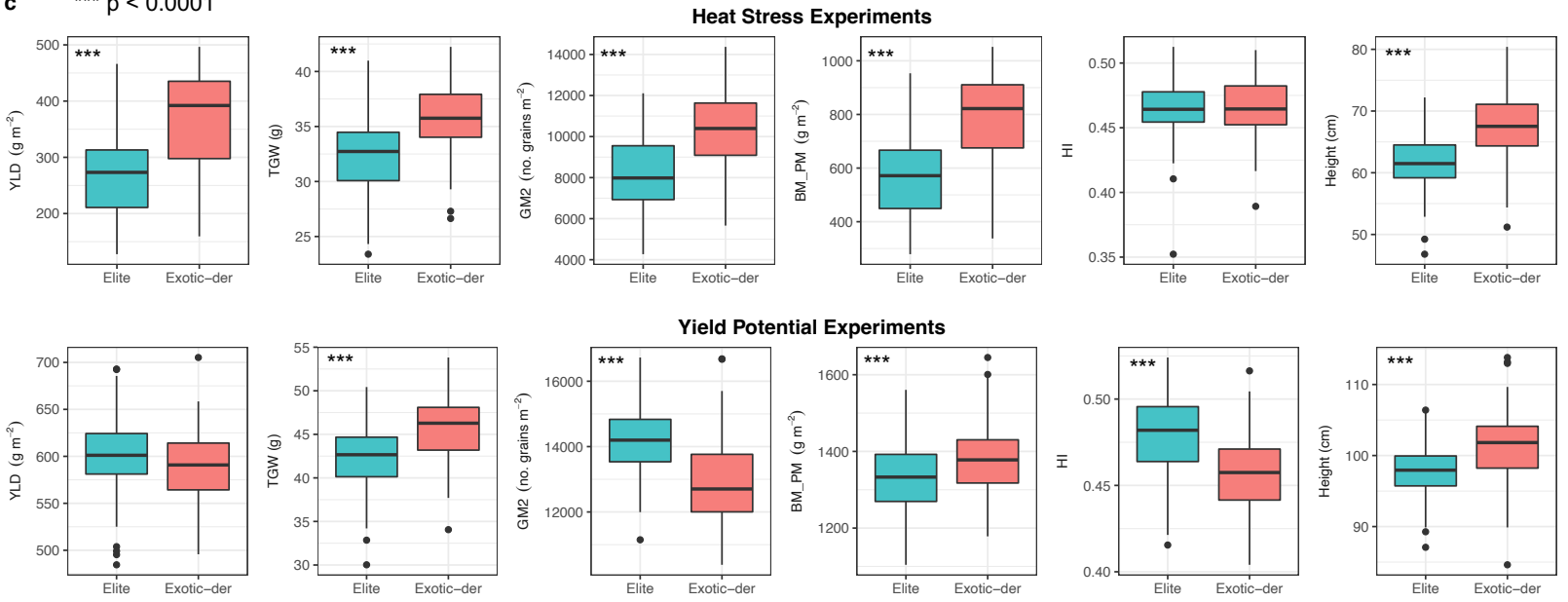

Figure 1. Physiological assessment of HiBAP I panel, comparing elite and exotic-derived lines under heat stressed and yield potential conditions. a) Number of lines from each group b) Effect of heat stress on traits, showing the percentage difference compared to yield potential conditions. c) Comparison of yield (YLD), thousand grain weight (TGW), grain number (GM2), biomass at physiological maturity (BM_PM), harvest index $(\mathrm{HI})$ and plant height $(\mathrm{PH})$ between elite and exotic-derived lines in HiBAP I measured under both heat stress and yield potential conditions. The significance of the differences between elite and exotic-derived lines for each trait was assessed using two-tailed $t$ tests with no assumption of equal variance. $p$-values below 0.01 were considered significant $\left({ }^{*}\right)$, below 0.001 very significant $\left(^{* *}\right)$ and below 0.0001 highly significant $\left({ }^{* * *}\right)$.

Heat stress was imposed by delayed sowing compared to the check environment (Figure S1) and, across both years of evaluation, this reduced yield by $48.1 \%$ and shortened the crop cycle duration by more than $30 \%$ (Fig. 1b, Table S3). When we analysed the response to heat stress of the lines based on their pedigree, exotic-derived lines exhibited an average of $37.7 \%$ higher yield compared to elite lines under heat stressed conditions (Fig. 1c, upper). Biomass, the trait most affected by heat stress, was $39 \%$ 
higher in exotic-derived lines, and other yield components, except for harvest index ( $\mathrm{HI})$, were significantly higher in exotic-derived lines than elite lines (Fig. 1c, upper). Under yield potential conditions, exotic-derived lines did not show a yield penalty compared to elite lines, as was reported by (21) (Fig. 1c, lower). Exotic lines were on average $5.6 \mathrm{~cm}$ and $3.8 \mathrm{~cm}$ taller than elite lines under heat stressed and yield potential conditions, respectively. No differences in phenology were observed between the groups in either of the environments. Plant height was not correlated with yield under yield potential conditions $(r=-0.007)$, but positive correlations were observed between plant height and yield under heat stressed environments $(r=0.699)$. The better performance of exotic-derived lines was validated using stress susceptibility index (SSI). This measure is negatively correlated with yield under heat stressed conditions; thus, lower SSI values indicate higher tolerance to a stressful environment. Compared to elite lines, exotic-derived lines had significantly lower SSI values for yield, grains per $\mathrm{m}^{2}$ (GM2) and biomass at physiological maturity (BM_PM), but not for thousand grain weight (TGW) (Table 1).

Table 1. Stress susceptibility index (SSI) calculated for yield (YLD), thousand grain weight (TGW), grains per $\mathrm{m}^{2}$ (GM2) and biomass at physiological maturity (BM_PM) of elite and exotic-derived lines obtained from adjusted means for two years of data in each environment. $r_{p}$ corresponds to the phenotypic correlation with the yield obtained under heat environments. Data represents the mean \pm S.D.

\begin{tabular}{|c|c|c|c|c|c|}
\hline Trait & $r_{p}$ (YLD_Heat) & Elite & & Exotic & \\
\hline & & $n=83$ & & $n=66$ & \\
\hline SSI_YLD & -0.976 & $1.16 \pm 0.24$ & a & $0.79 \pm 0.32$ & b \\
\hline SSI_TGW & -0.439 & $1.03 \pm 0.2$ & a & $0.95 \pm 0.27$ & $a$ \\
\hline SSI_GM2 & -0.951 & $1.26 \pm 0.42$ & a & $0.60 \pm 0.52$ & b \\
\hline SSI_BM_PM & -0.946 & $1.13 \pm 0.20$ & a & $0.85 \pm 0.23$ & b \\
\hline
\end{tabular}

Letters indicate the statistical significance between Elite and Exotic groups.

Means followed by different letters are significantly different $(p$-value $<0.01)$

according to pairwise $t$ tests.

Additional physiological traits were measured in the experiments to help understand the physiological basis of the superiority of the exotic-derived lines under heat stressed conditions. Exotic-derived lines had significantly higher normalised difference vegetative index (NDVI), a proxy for biomass, and significantly lower canopy temperature (CT) during both vegetative stage and grain filling stages under heat stressed conditions but not under yield potential conditions (Fig. 2). NDVI measured during vegetative and grain filling stages was positively correlated with yield (Fig. 2) while CT was negatively correlated with yield at both stages (Fig. 2). These correlations were present under heat stressed conditions but not under yield potential conditions. The correlations were steeper for exotic-derived lines than elite lines for both traits across both phenological stages, suggesting that NDVI and CT is having a higher impact on yield in exotic-derived lines compared with elite lines. Under heat stressed environments, both NDVI and CT presented similar correlations with BM_PM, GM2 and other yield components (Table S4) but no 
correlation was observed under yield potential. The SSI index calculated for yield was negatively correlated with agronomic and physiological traits except for $\mathrm{CT}$, where positive correlations were observed indicating that more tolerant lines had consistently cooler canopies (Table S4).

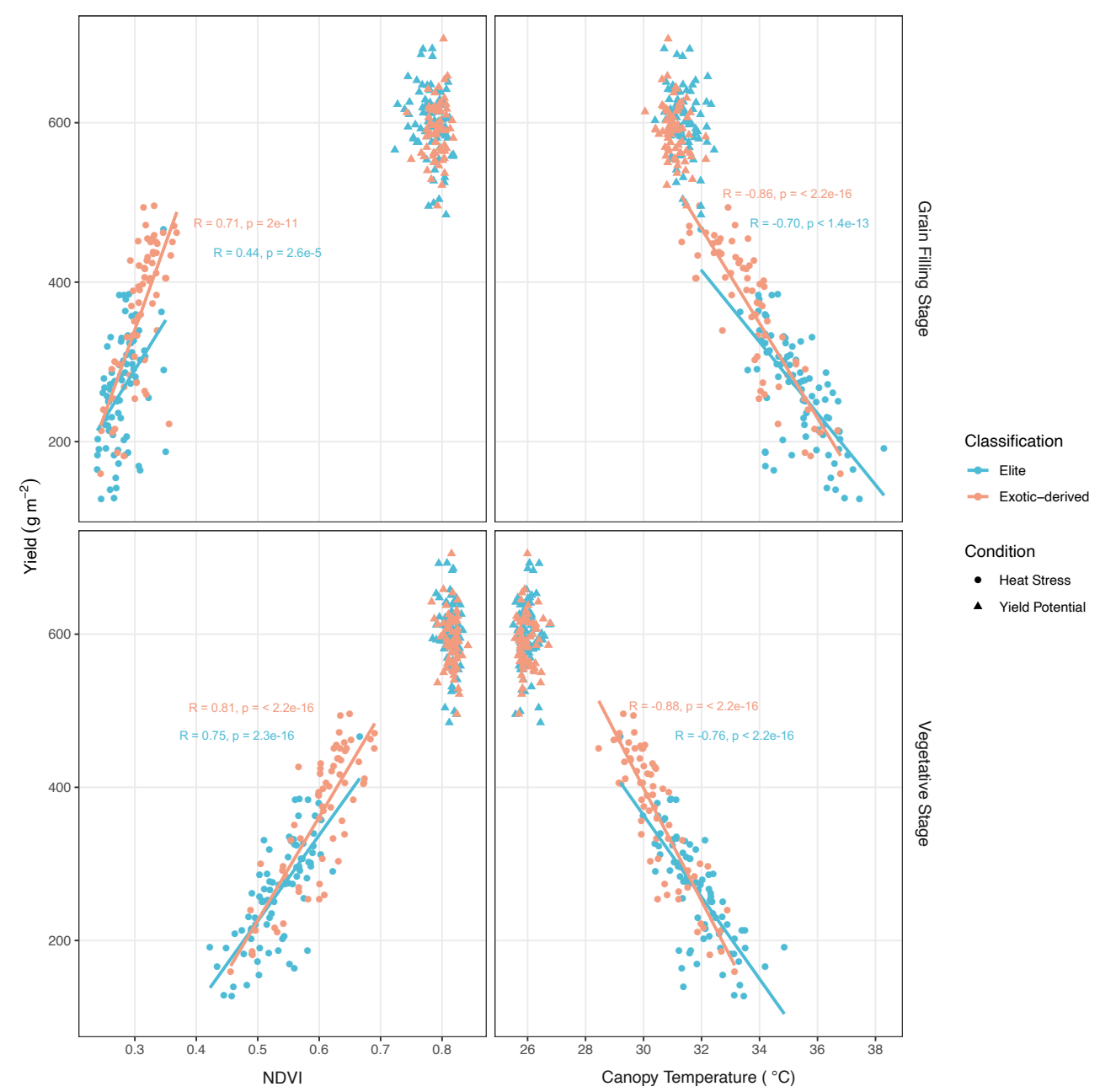

Figure 2. Relationship between normalized difference vegetation index (NDVI) and yield and between average canopy temperature (CT). NDVI and CT were measured with UAVs at pre-heading (vegetative stage) and during grain filling. Regression lines were added for groups with a significant correlation ( $p$ value $<=0.01$ ) calculated using Pearson's correlation coefficient.

\section{Genome-wide association analysis reveals genetic associations under heat stress}

To explore the genetic bases of these exotic-derived heat tolerance traits, marker-trait association analyses were performed using BLUE (Best Linear Unbiased Estimator) means from two or four replicates for each measured trait over two growing seasons. The most relevant MTAs are shown in Table S5, and all Manhattan plots are shown in Figure S2. We found 3 pleiotropic markers (Fig. 3a) on chr1B (chr1B-63398861: C), chr2B (chr2B-820002: C) and chr6D (chr6D-6276646: T). These MTAs were associated with all three heat stress indices along with multiple yield traits, including yield and canopy 
temperature, at both vegetative and grain filling stages, and were not associated with $\mathrm{HI}$ or phenology

(Fig. 3c, Fig S2).

a

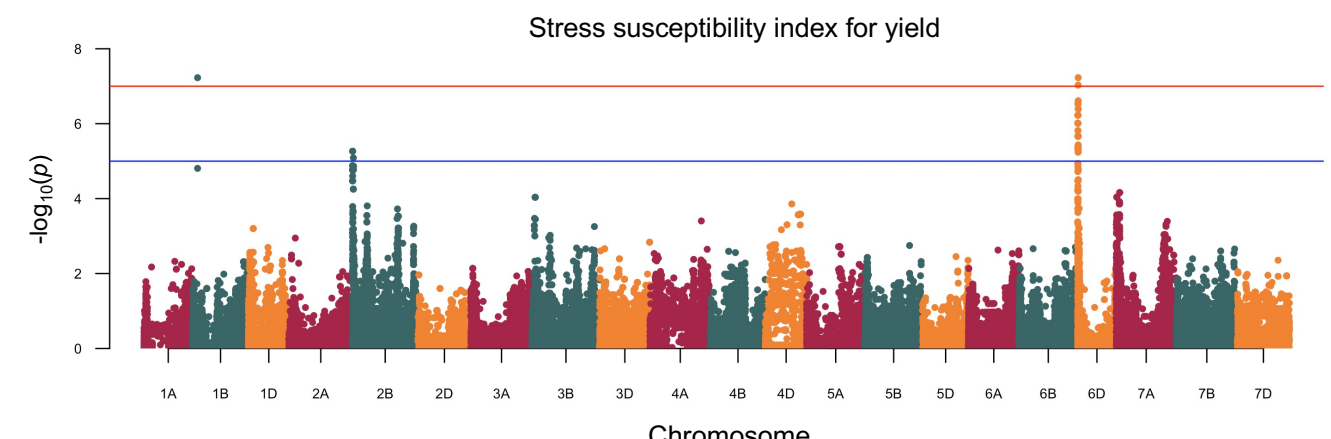

b
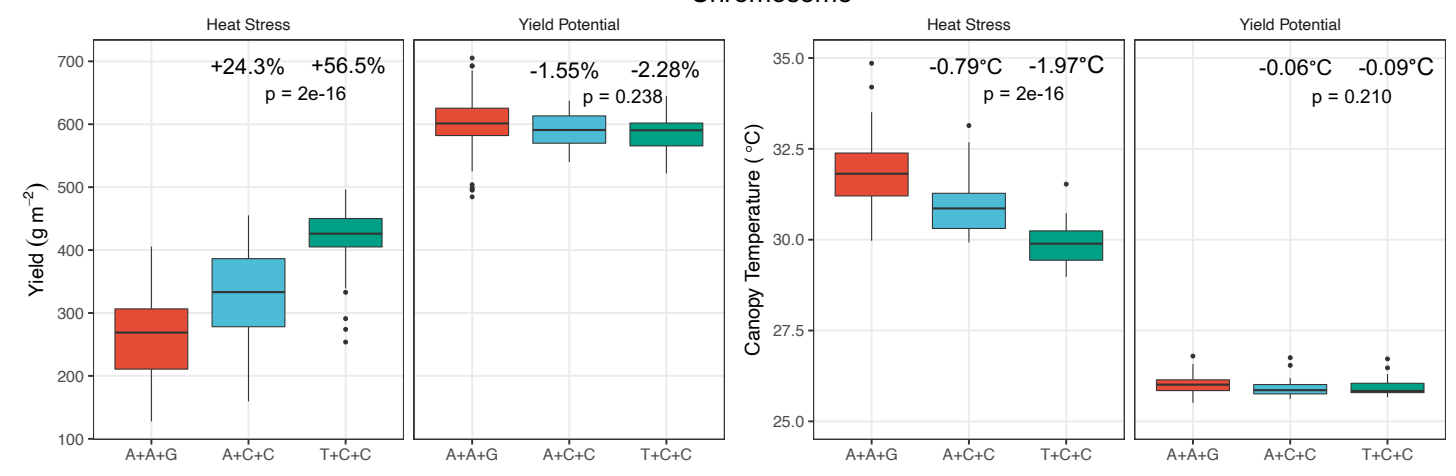

C

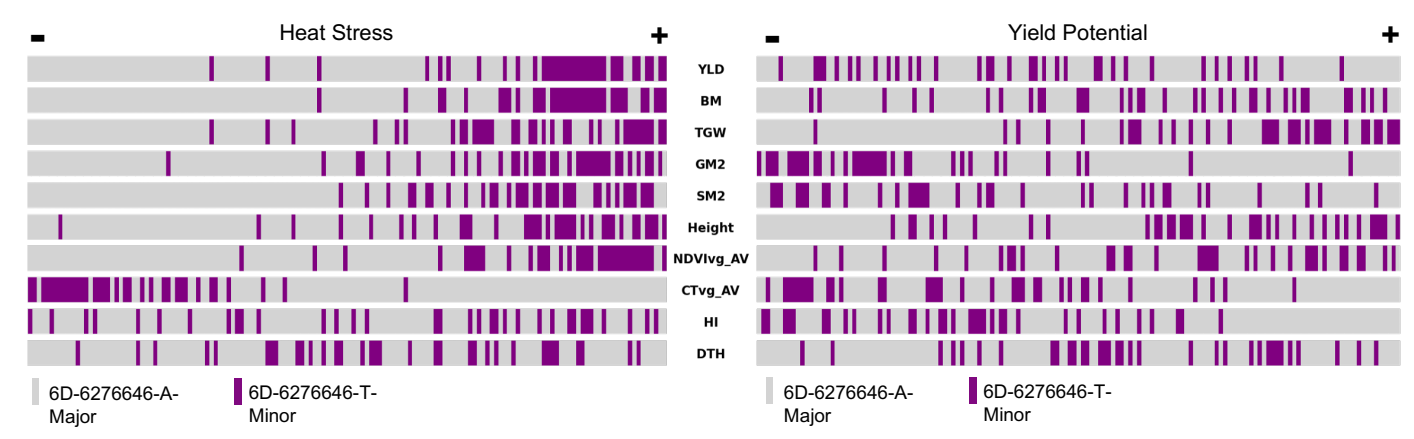

Figure 3. Genome-wide association study reveals genetic markers underlying heat tolerance traits

a) Manhattan plot showing the marker trait association for stress susceptibility index (SSI) for yield b) Specific marker allelic variants effects on yield and on canopy temperature under heat stress in chromosomes 6D (chr6D-6276646: T), 1B (chr1B-63398861: C) and 2B (chr2B-820002). The percentage change and ${ }^{\circ} \mathrm{C}$ change is calculated compared to lines with the major alleles at all three MTAs.

Significance of allele combinations was computed using a one-way ANOVA test c) Phenotype distribution under heat stress and yield potential conditions highlighting the rank of $6 \mathrm{D}$ minor allele carriers for each phenotype where lines in the panel are ordered from lowest to highest value for each trait. 
Lines with the minor $\mathrm{C}$ allele on $1 \mathrm{~B}$ and $2 \mathrm{~B}$ and the major $\mathrm{A}$ allele on $6 \mathrm{D}$ have $24.3 \%$ higher yield under heat stress; lines which also have the minor T allele on $6 \mathrm{D}$ have $56.7 \%$ higher yield under heat stress compared to lines with the three major alleles (Fig. 3b). Assuming the three alleles do not interact epistatically, the $\mathrm{T}$ allele on $6 \mathrm{D}$ can be estimated to increase yield under heat stress by $32.4 \%$. Lines with the minor allele at all three MTAs show a reduction in canopy temperature of $1.97^{\circ} \mathrm{C}$ and $2.37^{\circ} \mathrm{C}$, at vegetative and grain filling stages, respectively, when compared to lines with the major allele at all three positions (Fig. 3b). Under yield potential conditions, no difference was observed between minor and major allele combinations for yield or for canopy temperature (Fig. 3b). The minor allele at each of these MTAs is predominantly found in exotic-derived lines with 50/55 (1B), 44/45 (2B) and 33/33 (6D) lines with the minor allele classified as exotic-derived (Table S2).

\section{Aegilops tauschii introgression underlies 6D MTA}

Due to the better performance of exotic-derived lines under heat stress and exotic-derived lines possessing alleles for heat tolerance, we searched for introgressed material overlapping the MTAs. We detected introgressed material in HiBAP I lines by looking for regions with reduced mapping coverage and SNPs shared with Ae. tauschii, Th. ponticum or S. cereale but not with wheat cultivars Weebil, Pavon76 or Norin61. We identified introgressed Ae. tauschii material at the beginning of 6D in all 33 lines with the T/T genotype and all 7 lines with the A/T genotype. Multiple offspring of the same Sokoll Weebil1 cross show that recombination occurs readily within the segment, breaking up the segment into variable sizes (Fig. S3). The full-length, unbroken segment is 31.6Mbp in length, as seen in Sokoll (HiBAP_57) (Fig. 4a). By comparing the overlapping segments between lines, we found a 5.05Mbp and 6.85Mbp that is present in all lines with the $T / T$ or $A / T$ genotype at $6 D-6276646$ and absent in all the lines with the $A / A$ genotype (Fig. 4a). In A/T lines, the introgression itself appears to be heterozygous, evidenced by intermediate mapping coverage deviation compared to the homozygous lines and by heterozygous SNPs matched to Ae. tauschii. Using chromosome and protein alignments, we anchored this core region to the Ae. tauschii reference genome, Aet $\mathrm{v} 4.0$ (26), and extracted the syntenic 1.49Mbp region between 4.63Mbp and 6.12Mbp that likely contains the gene(s) responsible for the MTA (Fig. 4b, 4c). We found no evidence of introgressed material overlapping the $1 \mathrm{~B}$ or $2 \mathrm{~B}$ MTAs. 


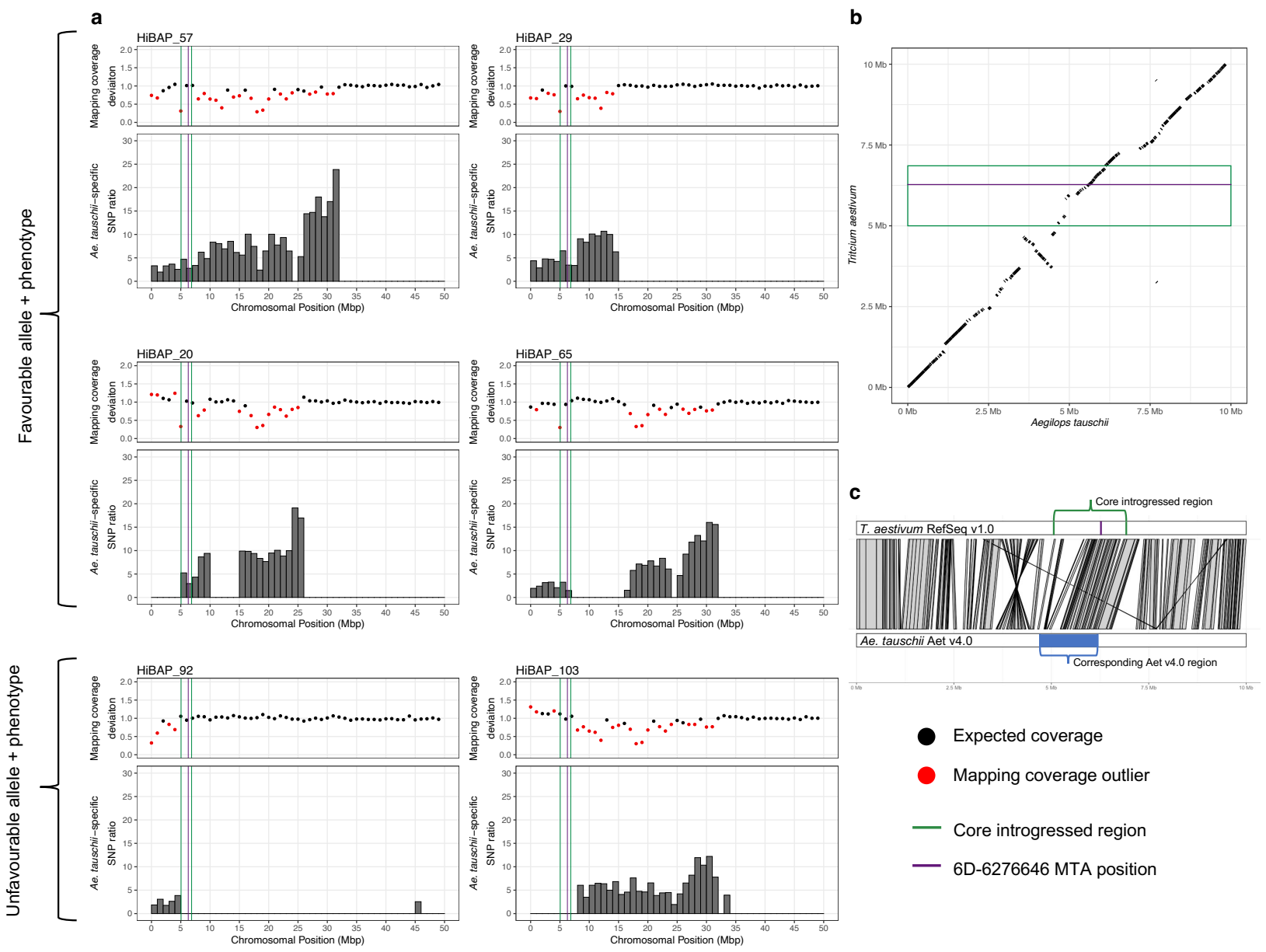

Figure 4. Aegilops tauschii introgression underlies chr6D-6276646 MTA. a) Visualising Ae. tauschii introgressions across the first $50 \mathrm{Mbp}$ of chr6D in six HiBAP I lines, four containing the favourable allele at chr6D-6276646 (HiBAP 57, 29, 48, and 65) and two containing the unfavourable A allele at chr6D6276646 (HiBAP 92 and 103). Mapping coverage deviation was computed between the HiBAP line and the median of the panel in $1 \mathrm{Mbp}$ windows. Red points are statistically significant outliers. Ae. tauschiispecific SNP ratio in each $1 \mathrm{Mbp}$ window was calculated by dividing the number of homozygous $A e$. tauschii-specific SNPs in that window by mean number of homozygous Ae. tauschii-specific SNPs in that window across the panel. Green lines mark the borders of the region common to all lines with the $T$ genotype, corresponding to a $1.80 \mathrm{Mbp}$ region in CS and a $1.49 \mathrm{Mbp}$ in Ae. Tauschii. The purple line indicates the MTA position. b) Synteny between 6D:1-10,000,000 in CS RefSeq v1.0 (27) and Ae. tauschii Aet v4.0 (26). The green box indicates the $1.80 \mathrm{Mbp}$ region (1.49Mbp relative to Ae. tauschii) common to all lines with $\mathrm{T}$ genotype, corresponding to the green region in part $a$. The purple line indicates the MTA position c) Alignment of 6D:1-10,000,000 in CS RefSeq v1.0 (27) and Ae. tauschii Aet v4.0 (26), illustrating how the orthologous region in Ae. tauschii was identified and extracted. 


\section{Candidate genes for MTAs in 1B, 2B and 6D}

For the 6D MTA, we identified the syntenic region in the Ae. tauschii genome and a list of genes that had been introgressed. As we are unaware of the Ae. tauschii accession that has been introgressed, we also looked at the genes within the orthologous region in four other available chromosome-level Ae. tauschii assemblies (28). Between accessions, this region varies between 1.49Mbp and $1.82 \mathrm{Mbp}$ in length from and contains between 26 and 33 genes (Table S6). These include a MIKC-type MADS-box gene orthologous to SUPPRESSOR OF OVEREXPRESSION OF CONSTAN 1 (SOC1); a mitogen-activated protein kinase (MAPK) gene found in two of the Ae. tauschii accessions with no orthologue in wheat; and a pair of type-B two-component response regulator receiver proteins, orthologous to type-B Arabidopsis response regulators (ARRs) with closest similarity to ARR-11. One member of the pair, AET6Gv20025700, appeared to have a myb-binding domain that is missing from the wheat orthologue gene model. However, after manual reannotation, we found that this difference was a misannotation in wheat so likely not causing a functional difference. We also found that both ARR genes were expressed specifically in spike and grain in both Ae. tauschii and wheat, whereas the other candidate genes were expressed across leaf, root, spike and grain tissues. For the 1B and 2B MTAs, as they were not within an introgression, we submitted the sequence $1 \mathrm{Mbp}$ up and downstream of the MTA to Knetminer, a gene discovery tool (29). Within the 2B interval, we 10identified DEHYDRATION-RESPONSIVE ELEMENTBINDING PROTEIN 1A (DREB1A) and STEROL GLUCOSYLTRANSFERASE (SGT) as promising candidate genes.

\section{Discussion}

\section{Exotic-derived lines have higher heat tolerance with no yield penalty under favorable conditions}

Exotic parents are routinely used to increase genetic diversity in wheat pre-breeding pipelines and their enhanced performance has been demonstrated under salinity $(30)$, drought $(14,19)$ and heat stress $(18$, 31). In the present study, exotic-derived lines performed better under heat stress than elite lines with no yield penalty under yield potential conditions. This increased yield under heat stress was associated with a range of factors, including higher biomass throughout the crop cycle, higher grain number and cooler canopy temperature during both vegetative and grain filling stages. Cooler canopies have been previously associated with higher tolerance to drought and heat irrigated environments (32) and with optimised root distribution in bread wheat (33). Plants with an optimized root system are able to satisfy the high evaporative demand through elevated transpiration rates under hot irrigated conditions and thus maintain cooler canopies (34). Higher transpiration rates are associated with increased stomatal conductance that, in turn, is associated with higher photosynthesis that can explain the higher biomass observed in exoticderived lines in comparison with elite lines. However, according to temperature response models in wheat (6), the observed reduction in plant temperature of approximately $2^{\circ} \mathrm{C}$ would be unlikely to account alone for the $>50 \%$ increased yield of exotic lines according to temperature response models in wheat (6). 
Despite variation in plant height being restricted, exotic-derived lines were taller than elite lines in both environments. Taller plants have a better light interception and light distribution, in comparison with shorter genotypes, and this has been associated with increased photosynthesis (35). Therefore, plant height can also influence the better performance of exotic-derivatives. Among all stress indices, the stress susceptibility index (SSI) is thought to be the most useful index for evaluating tolerant cultivars. Exoticderived lines had significantly lower SSI than elite lines, adding additional support to the resilience of this exotic material under heat stress.

\section{Combining high density genotyping and high-throughput phenotyping to uncover novel markers for heat stability}

The value of de novo SNP discovery through sequencing in breeding efforts is starting to be more widely recognised. In conjunction with high throughput phenotyping methods (36), high density, unbiased markers can be leveraged to discover novel MTAs or to narrow pre-existing QTL intervals to provide more robust markers for global breeding programs $(37,38)$. Using these methods, we have identified three pleiotropic markers on chromosomes $1 \mathrm{~B}, 2 \mathrm{~B}$ and $6 \mathrm{D}$ that when stacked increase yield by $56.5 \%$ and reduce canopy temperature by $1.97^{\circ} \mathrm{C} / 2.37^{\circ} \mathrm{C}$ under heat stress conditions when compared to lines containing the three major alleles at these positions (Fig. 3b). These markers were associated with multiple agronomically important traits under heat stress including yield, grain per square meter, grain filling rate and biomass (Fig. S2). Despite being in apparently disparate regions of the genome, the 1B and $2 \mathrm{~B}$ minor alleles always occur together and the $6 \mathrm{D}$ minor allele usually occurs with the $1 \mathrm{~B}$ and $2 \mathrm{~B}$ minor alleles. This suggests that there may be functional linkage between the markers. All three MTAs are predominantly found in exotic-derived lines but are not exclusive to any of the exotic categories as we see them in synthetic, introgression line and landraces derivatives. This brings their origin into question as their most recent pedigree suggests that the favorable alleles have come from different sources.

\section{Aegilops tauschii introgression underlies heat tolerance MTA}

By utilising mapping coverage information and species-specific SNPs, we identified that the MTA on 6D was within an Ae. tauschii introgression. We show that this introgression readily recombines within CIMMYT germplasm by comparing the introgressed segment in different offspring off the same cross. Due to concerns regarding linkage drag and lack of recombination of wild relative introgressions (38), this is promising for the deployment of introgressed segments from the primary genepool into breeding programmes. The recombination enabled us to reduce the size of the interval responsible for the MTA by looking for the region always present in lines with the favourable genotype. This is a novel technique to employ downstream of a GWAS, akin to hapmapping or in silico genetic mapping. The smallest segment in the panel that contains the MTA is around $5 \mathrm{Mbp}$ and can likely be broken down further; the small size and it's telomeric location make it amenable for deployment in breeding programmes. 
The longest unbroken segment is present in Sokoll, a commonly used advanced synthetic-derived line. Recombination within the segment takes place in all Sokoll $\times$ Weebil1 crosses yet is unbroken in Sokoll. Therefore, Sokoll may be the donor line for this marker in many of the lines in HiBAP I. This would make sense given its presence in many of the pedigree histories of CIMMYT's synthetic derived lines (Table S2). Some of the HiBAP I lines contain an Ae. tauschii segment that contains both the 6D MTA and a resistance gene upstream that underlies an MTA from a recent GWAS in Ae. tauschii (39). If the accession of Ae. tauschii in the HiBAP I lines confers the same resistance these lines could be used as donors for both traits.

The 6D MTA uncovered is supported by an MTA for heat tolerance reported in $(13,22)$ nearby on 6D. Singh et al., 2018 (13) state that the 6D MTA overlapped with an Ae. tauschii introgression, using speculative markers and pedigree-based inference. Here, we confirm this speculation and then demonstrate its ability to recombine and narrow down the introgressed region conferring the heat resilient phenotype through in silico introgression mapping.

\section{Putative candidate genes underlying heat stability}

Following the identification of the core introgressed region, we extracted the syntenic orthologous region from five Ae. tauschii chromosome-level assemblies and used these as our source for putative candidate genes rather than the wheat reference genome. When appropriate, using non-reference genomes is important because the variation underlying the trait of interest might be absent from the reference genome. To select candidate genes for the observed yield stability under heat stress, we carried out extensive literature searches on the introgressed Ae. tauschii genes or the wheat genes within the interval (for the 1B and 2B MTAs); this uncovered several new candidate genes for further dissection.

We found two type-B two-component response regulator genes, with members of this family acting as transcription factors in the cytokinin signalling pathway (40). They have been linked to photoperiod stress protection via root-derived cytokinins (41) and negative regulation of drought response (42) via root cytokinin pathways (43); the resulting elevated levels of cytokinin have been linked to heat stress tolerance (44). The orthologues do not have identical protein sequences which could underlie a functional difference. However, these genes are expressed only in spike and grain in Chinese Spring and $A e$. tauschii (if this holds following introgression) which may make these genes unlikely to be involved in the heat tolerant phenotype as it is established during the vegetative stage and maintained through grain filling. We also found a MIKC-type MADS-box transcription factor orthologous to the Arabidopsis gene SOC1, overexpression of which leads to chloroplast biogenesis, elevated photosynthesis, and tolerance to prolonged heat stress (45). Despite the protein sequences of the Ae. tauschii and wheat orthologues being identical, introgressed genes are often expressed differently when placed in a different genomic background (46) and upregulation of this gene may lead to the heat tolerant phenotype seen in SOC1 overexpression lines (45). Finally, we identified a novel MAPK gene. MAPKs play important roles in 
regulating responses to abiotic stress. They have been linked to oxidative stress tolerance under heat stress in wheat (47) and are involved in the heat stress response in Arabidopsis, maize and rice (48). The novelty of this gene and the presence-absence variation between Ae. tauschii accessions suggests this gene is recently evolved and possibly involved in environmental adaptation. The gene DREB1A was identified within the chr2B-820002 interval; this gene is part of a family of plant-specific transcription factors that bind DRE/CRT elements in the response to abiotic stresses. Overexpression of DREB1 in wheat led to drought tolerance and increased photosynthetic efficiency (49) and DREB2 overexpression lines displayed cold and heat tolerance (50). The 2B interval also contained STEROL

GLUCOSYLTRANSFERASE (SGT) which has been identified to affect heat stability in both knockout and overexpression studies in Arabidopsis $(51,52)$.

Our proposed candidate genes for the 6D MTA differ from those proposed by Singh et al., 2018 (13). By using the Ae. tauschii genomes rather than relying solely on the wheat reference genome, we have demonstrated that the isoflavone reductase gene they proposed is not present in the core introgressed region. The other candidates were possibly disregarded due to lack of non-synonymous mutations identified from their markers; however, this ignores the possibility of differences in expression underlying a functional difference. These differences highlight the value of considering non-reference genomes downstream of a GWAS, particularly when divergent material has been introduced and the phenotype may be non-allelic in nature.

\section{Implications for breeding for climate change}

These three markers can be deployed into marker-assisted breeding or introgression pipeline programmes to incorporate heat resilience traits into elite cultivars. The fact that no yield penalty was identified under more favourable conditions adds value to their deployment, especially given the negative impact that has been documented in terms of yield stability under increasing temperatures using extensive international data (53). The donor lines for these markers will be selected using our introgression mapping approach to introduce minimal linkage drag alongside the traits of interest.

\section{Materials and Methods}

\section{Plant material and Growth Conditions}

The High Biomass Association Mapping Panel HiBAP I consisted of 149 spring wheat lines (Table S2) and is composed of elite high yielding lines and lines with exotic material in their pedigree history. These exotic lines include primary synthetic derivative lines; Mexican and other origin landraces derivatives and elites containing introgressions, such as from Thinopyrum ponticum and Secale cereale. All lines have agronomically acceptable backgrounds and a restricted range of maturity and height to avoid extreme phenology or height confounding the expression of biomass and other traits. HiBAP I was evaluated 
during 2015/16 and 2016/17 under yield potential (referred to hereafter as YP16 and YP17, respectively) and heat stressed conditions ( $\mathrm{Ht} 16$ and $\mathrm{Ht} 17)$. Heat stressed conditions were created with delayed sowing where emergence was registered in March instead of November or December as in a normal growing cycle (Table S1, Fig. S1).

The field experiments were carried out at IWYP-Hub (International Wheat Yield Partnership Phenotyping Platform) situated at CIMMYT's Experimental Station in Campo experimental Norman E. Borlaug (CENEB) in the Yaqui Valley, near Ciudad Obregon, Sonora, Mexico (27²4' N, 10956' W, 38 masl) under fully irrigated conditions for both yield potential and heat stressed experiments. The soil type at the experimental station is a coarse sandy clay, mixed montmorillonitic typic caliciorthid. It is low in organic matter and is slightly alkaline ( $\mathrm{pH}$ 7.7) (54). Experimental design for all environments was an alpha-lattice. Yield potential experiments consisted of four replicates in raised beds ( 2 beds per plot each $0.8 \mathrm{~m}$ wide) with four (YP16) and two (YP17) rows per bed $(0.1 \mathrm{~m}$ and $0.24 \mathrm{~m}$ between rows respectively) and $4 \mathrm{~m}$ long. For heat stressed experiments, two replicates were evaluated for HiBAP I in $2 \mathrm{~m} \times 0.8 \mathrm{~m}$ plots with three rows per bed (Table S1). Seeding rates were $102 \mathrm{Kg} \mathrm{ha}^{-1}$ and $94 \mathrm{Kg} \mathrm{ha}^{-1}$ for YP and $\mathrm{Ht}$ experiments, respectively. Appropriate weed disease and pest control were implemented to avoid yield limitations. Plots were fertilised with $50 \mathrm{~kg} \mathrm{~N} \mathrm{ha}^{-1}$ (urea) and $50 \mathrm{~kg} \mathrm{P} \mathrm{ha}^{-1}$ at soil preparation, $50 \mathrm{~kg} \mathrm{~N} \mathrm{ha}^{-1}$ with the first irrigation and another $150 \mathrm{~kg} \mathrm{~N} \mathrm{ha}^{-1}$ with the second irrigation. Rainfall, radiation, maximum, minimum and mean temperature by month for all the years of evaluation are presented in Fig. $\mathbf{S 1 .}$

\section{Agronomic measurements}

Phenology of the plots was recorded during the cycle using the Zadoks growth scale (GS) (55), following the average phenology of the plot (when $50 \%$ of the shoots reached a certain developmental stage). The phenological stages recorded were heading for heat experiments (GS55, DTH), anthesis for yield potential experiments (GS65, DTA) and physiological maturity (GS87, DTM) for both experiments. Percentage of grain filling (PGF) was calculated as the number of days between anthesis and physiological maturity divided by DTM.

Plant height was measured as the length of five individual shoots per plot from the soil surface to the tip of the spike excluding the awns. Spike, awn and peduncle length were measured in five shoots per plot before physiological maturity (PM). Fertile $\left(\mathrm{SPKLSP}^{-1}\right)$ and infertile spikelets per spike $\left(\mathrm{InfSPKLSP}^{-1}\right)$ were also counted in five spikes per plot at PM.

At physiological maturity, grain yield and yield components were determined using standard protocols (56). Samples of 100 (YP16), 50 (YP17) or 30 (Ht16, Ht17) fertile shoots were taken from the harvested area at physiological maturity to estimate yield components. The sample was oven-dried, weighed and threshed to allow calculation of harvest index $(\mathrm{HI})$, biomass at physiological maturity (BM_PM), spikes per square meter (SM2), grains per square meter (GM2), number of grains per spike (GSP) and grain weight 
per spike (GWSP). Grain yield was determined on a minimum of $3.2 \mathrm{~m}^{2}$ to a maximum of $4.8 \mathrm{~m}^{2}$ under yield potential experiments and $1.6 \mathrm{~m}^{2}$ under heat experiments. In yield potential experiments only, to avoid edge effects arising from border plants receiving more solar radiation, $50 \mathrm{~cm}$ of the plot edges were discarded before harvesting. From the harvest of each plot, a subsample of grains was weighed before and after drying (oven-dried to constant weight at $70^{\circ} \mathrm{C}$ for $48 \mathrm{~h}$ ) and the ratio of dry to fresh weight was used to determine dry grain yield and thousand grain weight (TGW). GM2 was calculated as (Yield/TGW) $\times$ 1000. BM_PM was calculated from yield/HI. SM2 was calculated as BM_PM/(DW shoots/Shoot number).

\section{Unmanned Aerial Vehicle (UAV) for CT and NDVI estimation}

Aerial measurements data for CT and NDVI was collected using different aerial platforms. Each year, the logistics and availability determined which platform could be used for measuring the heat trials. A summary of the platforms used, together with the cameras and the achieved resolutions, is presented in Table S7. The multispectral and thermal cameras were calibrated onsite by measuring over calibration panels placed on the ground before and after each mission. An exception were the aircraft missions, where a calibration performed at the airfield would not be representative of the trial conditions. The flights were designed as a regular grid of north-south flightpaths covering the whole trial with images that overlapped $75 \%$ in all directions to ensure a good reconstruction of the orthomosaic. The flights were performed under clear sky conditions at solar noon \pm 2 hours.

NDVI and CT orthomosaics were obtained from the aerial images using the software Pix4D (need to confirm details). The orthomosaics were then exported to ArcGIS where a grid of polygons representing each polygon was adjusted on top of the image. To avoid the border effect, the polygons were buffered $0.5 \mathrm{~m}$ from the north and south border of the plot. Finally, the pixel values were extracted using the 'raster' package in R. We extracted the value of all the pixels enclosed within each polygon and removed possible outliers and calculated the average per plot.

\section{Stress Tolerance Indices}

To determine the effect of heat stress in the genotypes evaluated across years and panels, Stress susceptibility index (SSI) was calculated using data from yield potential (Yyp) and heat stressed (Yht) experiments. as:

$$
S S I=\frac{1-\frac{Y h t}{Y y p}}{1-\frac{\bar{Y} h t}{\bar{Y} y p}}
$$


where $\bar{Y}$ ht and $\bar{Y} y p$ are the mean yields of wheat lines evaluated under heat stress and yield potential conditions, respectively (57).

\section{Statistical analysis for phenotypic traits}

Data from both panels was analysed by using a mixed model for computing the least square means (LSMEANS) for each line across both years using the program Multi Environment Trial Analysis with $\mathrm{R}$ for Windows (METAR, (58)). When its effect was significant, DTA/DTH was used as a covariate (fixed effect) except for phenology. Broad sense heritability $\left(H^{2}\right)$ was estimated for each trait across both years as:

$$
H^{2}=\frac{\sigma_{g}^{2}}{\sigma_{g}^{2}+\frac{\sigma_{g e}^{2}}{e}+\frac{\sigma^{2}}{r e}}
$$

where $r=$ number of repetitions, $e=$ number of environments (years), $\sigma^{2}=$ error variance, $\sigma^{2} g=$ genotypic variance and $\sigma^{2} \mathrm{ge}=\mathrm{G} \times \mathrm{Y}$ variance. Unpaired $t$-tests for stress index (SSI) were conducted with the means across years to determine if the elite and exotic groups presented statistical differences with $p$ value $<0.001$.

\section{DNA extraction and Capture Enrichment and Genotyping}

All genotyping data relating to the HiBAP I panel in this paper was taken from (25). Briefly, flag leaf material from 10 plants per line was collected from field grown plots and pooled prior to extraction. DNA was extracted using a standard Qiagen DNEasy extraction preparation and quality and quantity assessed. From this DNA, dual indexed Trueseq libraries with an average insert size of $450 \mathrm{bp}$ were produced for each line and enriched using a custom MyBaits 12Mbp (100,000 120bp RNA probe) enrichment capture synthesised by Arbor Bioscience. Prior to enrichment, samples were pooled into 8 samples per capture reaction. Post enrichment libraries were sequenced using an S4 flowcell on an Illumina NovaSeq S6000 producing 150bp paired end reads. These reads were mapped to the RefSeq v1.0 Chinese Spring reference genome (27) using BWA mem (59) and following filtering as per (25), SNPs were called with BCFtools (60) and subsequently filtered using GATK. SNPs were then annotated using SNPeff 4.3 (61). To create a set of shared SNPs for use in GWAS SNPs for all lines were combined and loci with more than $10 \%$ missing data and a minor allele frequency (MAF) below $5 \%$ were removed.

\section{Genome-Wide Association Study (GWAS)}

GWAS analysis was conducted using the MLM method implemented in GAPIT (62). The effects of hidden familial relatedness were mitigated using principal component analysis eigenvectors 1-10 or membership coefficient matrices for 3-8 assumed subpopulations deduced by STRUCTURE (63) as covariates in the model. The EMMA (64) method was followed to create a kinship matrix required by the MLM method. 
Each MTA flanking interval was deduced by identifying the SNP position furthest upstream and downstream from the highest associated SNP that was above the -log P threshold of 5.

\section{Identifying regions of divergence}

RefSeq v1.0 (27) was split into $\mathrm{n}$ genomic windows of size $1 \mathrm{Mbp}$ and $100 \mathrm{Kbp}$ using bedtools makewindows (65). Using the alignments produced in (25) and detailed above, the number of reads mapping to each window was computed using hts-nim-tools (66). To normalise by the sequencing depth of each line, read counts were divided by the number of mapped reads that passed the filters, producing normalised read counts $\mathrm{c}$. Different windows of the genome have variable mapping coverage rates, so to compute coverage deviation we must compare each window to the same window in the other lines in the collection. Median normalised read counts, $\mathrm{m}$, were produced, containing the median for each genomic window across the 149 lines. Mapping coverage deviation, $d$, was then defined for each line as:

$$
d_{i}=\frac{c_{i}}{m_{i} \cdot \varepsilon}
$$

for window $i \in\{1,2, \ldots, n\}$, where $\varepsilon$ is the median $d$ value across the genome for the line. Statistically significant $d$ values were calculated using the scores function from the $R$ package 'outliers' with median absolute deviation (MAD) and probability of 0.99 . This method was based on (67).

\section{Producing species-specific SNPs}

Paired-end whole-genome sequencing data for the Ae. tauschii reference accession AL8/78 (26) and 5 additional accessions that represent 5 different clades (28), 4 Secale cereale accessions (68), Secale. vavilovii (68), Thinopyrum ponticum (69), and T. aestivum cultivars Weebil (69), Norin61 (69) and Pavon76 (46) were mapped to RefSeq v1.0 (27), filtered and SNP called as described for the genotyping above and in (25). Homozygous SNPs were retained if they had between 10 and 60 reads supporting the alternative allele and an allele frequency $>=0.8$. Heterozygous SNPs were retained if they had oobi:between 10 and 60 reads mapped and were biallelic with each allele having >= 5 reads in support and an allele frequency $>=0.3$. SNPs from one relative species not shared with any of the other species or wheat cultivars were retained as species-specific SNPs. These species-specific SNPs were assigned to HiBAP SNPs if they matched in position and allele. Species-specific SNP ratios were calculated by dividing the number of SNPs in each window matched to a species-specific SNP by the mean number of SNPs matched to that species in that window across HiBAP I. SNP ratio scores below 1.45 were removed to keep enriched scores only. 


\section{Synteny between Ae. tauschii and T. aestivum}

The first $10 \mathrm{Mb}$ of $6 \mathrm{D}$ from CS and Ae. tauschii Aet v4.0 (26) were aligned using Minimap2 (70) with parameters $-x$ asm10. Alignments $<2.5 \mathrm{~Kb}$ in length or with mapping quality $<40$ were discarded. The dot plot was produced using pafr R package (71). Proteins encoded by genes in the first 10Mbp of 6D in $A e$. tauschii and CS were aligned using BLASTp (72). Protein alignments and minimap2 alignments were used to anchor either side of the region commonly introgressed in all lines with the 6D T genotype to anchor the region from CS to Ae. tauschii. The Ae. tauschii genes and their proteins within this segment are considered as candidate genes. BLASTp (72) was used to compare these proteins to wheat proteins. Protein domains were identified using HMMER hmmscan (73) via ebi using Pfam, TIGRFAM, Gene3D, Superfamily, PIRSF, and TreeFam databases.

\section{Extracting corresponding region and genes from Ae. tauschii genomes}

Proteins encoded by genes in the first $10 \mathrm{Mbp}$ of $6 \mathrm{D}$ in Ae. tauschii and CS were aligned using BLASTp (72). Protein alignments and minimap2 alignments were used to anchor either side of the region commonly introgressed in all lines with the $6 \mathrm{D} T$ haplotype to anchor the region from CS to $A e$. tauschii. The sequence extracted from the Ae. tauschii reference genome was aligned to the other 4 chromosome-level assemblies using minimap2 (70) with parameters -x asm5. Alignments below length 5000 or quality of 40 were removed. The coordinates of each orthologous region were determined manually and the genes within these coordinates extracted from the respective gff files. The Ae. tauschii genes and their proteins within these segments were considered as candidate genes for functional exploration. BLASTp (72) was used to compare these proteins to wheat proteins. Protein domains were identified analysed using HMMER hmmscan (73) via ebi using Pfam, TIGRFAM, Gene3D, Superfamily, PIRSF, and TreeFam databases. Novelty of genes was determined by aligning the extracted protein sequence to each genome using tblastn (72).

\section{Exploring functionality of candidate genes}

The genes in each identified interval (except for those in the 6D interval) were submitted to Knetminer (29). The knowledge networks created for each gene were then studied to identify links to the trait from which each MTA was deduced including their biochemical function and orthologous genes being linked in other organisms such as Rice and Arabidopsis thaliana. For the Ae. tauschii genes introgressed into the $6 \mathrm{D}$ interval, we conducted extensive literature searches to identify genes with links to heat stress response based on functional studies of related genes.

\section{Reannotating candidate gene and assessing tissue-specific expression}

To test whether the missing myb-binding domain in the TraesCS6D02G014900 annotation was real or an artefact, we manually reannotated the gene. We identified the exon containing the MYB-binding domain 
in the wheat orthologue by aligning the coding sequence from the tauschii orthologue to Chinese Spring RefSeq v1.0 (27) using tblastn (72). We mapped Chinese Spring RNAseq data from leaf, root and shoot to RefSeq v1.0 (27) using HISAT2 (74) and assembled transcripts using cufflinks (75). We visually inspected the coding sequencing and RNA alignments IGV (76), which showed that the MYB-binding domain exon is present and expressed in wheat. To check whether the protein has a premature stop codon that might have interfered with the annotation and translation of the myb-binding domain, we extracted the coding sequence from the assembled transcript and checked for the presence of a complete open reading frame with no stop codons using EMBOSS getorf (77). Finally, we checked the presence of intact domains with HMMER hmmscan (73) via ebi using Pfam, TIGRFAM, Gene3D, Superfamily, PIRSF, and TreeFam databases. To explore qualitative expression of candidate genes, we mapped Ae. tauschii RNAseq data from leaf, root, seedling and developing grain 10dd (PRJEB23317) as above and abundances were counted using StringTie (78), taking the mean transcripts per million (TPM) across the replicates. Qualitative expression of the CS orthologues was explored using Wheat Expression Browser (79) and the previously leaf, root and shoot RNAseq data mapped above.

\section{Data availability}

Publicly available sequencing data used in this study is available at the European Nucleotide Archive (ENA): Th. ponticum - SRR13484812; S. vavilovii: ERR505040, ERR505041, ERR505042; S. cereale accession Lo90: ERR504990, ERR504991, ERR504992; S. cereale accession Lo176: ERR505005, ERR505006, ERR505007; S. cereale accession Lo282: ERR505015, ERR505016, ERR505017; S. cereale accession Lo351: ERR505035, ERR505036, ERR505037; Ae. Tauschii accession XJ65: SRR13961980; Y173: SRR13962062; SX60: SRR13962012; AY29: SRR13961834; KU2832: SRR13961928; Y215: SRR13962048; Weebil1: PRJEB35709; Norin61: PRJNA492239; Pavon76: https://opendata.earlham.ac.uk/wheat/under license/toronto/Hall 2021-10-08 wheatxmuticum/PIP_2495/200812 A00478 0126 AHN5W3DRXX/A10948 1 1/; Ae. tauschii RNAseq data: PRJEB23317; $T$. aestivum cv. Chinese Spring RNAseq data: Root - SRP133837; SRR6799264; SRR6799265; Leaf SRR6799258; SRR6799259; SRR6799260; Spike - SRR6802608; SRR6802609; SRR6802610; SRR6802611.

Phenotypic data presented in this paper for the HIBAP I panel evaluated under yield potential and heat stressed environments can be found in the Dataverse CIMMYT Research Data Repository at https://data.cimmyt.org/dataset.xhtml?persistentld=hdl:11529/10548643

\section{Acknowledgements}

This research was supported by the International Wheat Yield Partnership (IWYP) and by the Sustainable Modernization of Traditional Agriculture (MasAgro) an initiative from the Secretariat of Agriculture and 
Rural Development (SADER) and CIMMYT. Foundation for Food and Agriculture Research under the Grant ID: DFs-19-0000000013. AH was supported by BBSRC Core Strategic Programme Grant (Genomes to Food Security) BB/CSP1720/1; AH and RJ was supported by the BBSRC Designing Future Wheat grant BB/P016855/1, BBS/E/T/000PR9783 (DFW WP4 Data Access and Analysis). AH and RJ were also supported by BBSRC/IWYP BB/N020871/1. BC was supported by the BBSRC funded Norwich Research Park Biosciences Doctoral Training Partnership grant BB/M011216/1.

\section{Author contributions}

$\mathrm{AH}, \mathrm{GM}$ and MPR conceived of the idea and designed the experiment. GM, FP, FJPC and CRA collected the field data. GM analysed the physiological data. RJ conducted the genome-wide association study and Knetminer searches. BC conducted introgression analysis and introgressed candidate gene searches. $\mathrm{BC}, \mathrm{GM}$ and RJ wrote the manuscript. AH and MPR were responsible for funding and supervision. All authors reviewed the manuscript.

\section{Competing interests}

The authors declare no competing interests

\section{References}

1. FAOSTAT (2021) (January 18, 2022).

2. J. Jägermeyr, et al., Climate impacts on global agriculture emerge earlier in new generation of climate and crop models. Nat. Food 2021 211 2, 873-885 (2021).

3. C. M. Cossani, M. P. Reynolds, Physiological traits for improving heat tolerance in wheat. Plant Physiol. 160, 1710-1718 (2012).

4. M. P. Reynolds, et al., An integrated approach to maintaining cereal productivity under climate change. Glob. Food Sec. 8, 9-18 (2016).

5. S. K. Wegren, Food Security and Russia's 2010 Drought. Eurasian Geogr. Econ. 52, 140-156 (2011).

6. S. Asseng, et al., Rising temperatures reduce global wheat production. Nat. Clim. Chang. 201452 5, 143-147 (2014).

7. C. Zhao, et al., Temperature increase reduces global yields of major crops in four independent estimates. Proc. Natl. Acad. Sci. U. S. A. 114, 9326-9331 (2017). 
8. M. Tester, P. Langridge, Breeding technologies to increase crop production in a changing world. Science (80-. ). 327, 818-822 (2010).

9. G. E. Hart, J. Dvorak, M.-C. Luo, Z.-L. Yang, H.-B. Zhang, Communicated by The structure of the Aegilops tauschii genepool and the evolution of hexaploid wheat. Theor App/ Genet 97, 657-670 (1998).

10. G. Charmet, Wheat domestication: Lessons for the future. C. R. Biol. 334, 212-220 (2011).

11. D. Sehgal, et al., Exploring and Mobilizing the Gene Bank Biodiversity for Wheat Improvement. PLoS One 10, e0132112 (2015).

12. J. J. Valkoun, Wheat pre-breeding using wild progenitors. Euphytica $20011191119,17-23$ (2001).

13. S. Singh, et al., Harnessing genetic potential of wheat germplasm banks through impact-orientedprebreeding for future food and nutritional security. Sci. Rep. 8 (2018).

14. M. Reynolds, F. Dreccer, R. Trethowan, Drought-adaptive traits derived from wheat wild relatives and landraces. J. Exp. Bot. 58, 177-186 (2007).

15. R. M. Trethowan, A. Mujeeb-Kazi, Novel Germplasm Resources for Improving Environmental Stress Tolerance of Hexaploid Wheat. Crop Sci. 48, 1255-1265 (2008).

16. R. Ortiz, et al., Climate change: Can wheat beat the heat? (2008) https:/doi.org/10.1016/j.agee.2008.01.019 (January 18, 2022).

17. H. Aberkane, et al., Evaluation of durum wheat lines derived from interspecific crosses under drought and heat stress. Crop Sci. 61, 119-136 (2021).

18. C. M. Cossani, M. P. Reynolds, Heat Stress Adaptation in Elite Lines Derived from Synthetic Hexaploid Wheat. Crop Sci. 55, 2719-2735 (2015).

19. M. S. Lopes, M. P. Reynolds, Drought Adaptive Traits and Wide Adaptation in Elite Lines Derived from Resynthesized Hexaploid Wheat. Crop Sci. 51, 1617-1626 (2011).

20. M. P. Reynolds, et al., Strategic crossing of biomass and harvest index-source and sinkachieves genetic gains in wheat. Euphytica 213, 1-23 (2017).

21. G. Molero, et al., Elucidating the genetic basis of biomass accumulation and radiation use efficiency in spring wheat and its role in yield potential. Plant Biotechnol. J. 17, 1276-1288 (2019). 
22. S. Singh, et al., Direct introgression of untapped diversity into elite wheat lines. Nat. Food 2021 210 2, 819-827 (2021).

23. M. Reynolds, et al., Raising yield potential in wheat. J. Exp. Bot. 60, 1899-1918 (2009).

24. S. McCouch, et al., Mobilizing Crop Biodiversity. Mol. Plant 13, 1341-1344 (2020).

25. R. Joynson, et al., Uncovering candidate genes involved in photosynthetic capacity using unexplored genetic variation in Spring Wheat. Plant Biotechnol. J. 19, 1537-1552 (2021).

26. M. C. Luo, et al., Genome sequence of the progenitor of the wheat $D$ genome Aegilops tauschii. Nat. 20175517681 551, 498-502 (2017).

27. R. Appels, et al., Shifting the limits in wheat research and breeding using a fully annotated reference genome. Science 361 (2018).

28. Y. Zhou, et al., Introgressing the Aegilops tauschii genome into wheat as a basis for cereal improvement. Nat. Plants 2021 76 7, 774-786 (2021).

29. K. Hassani-Pak, et al., KnetMiner: a comprehensive approach for supporting evidence-based gene discovery and complex trait analysis across species. Plant Biotechnol. J. 19, 1670-1678 (2021).

30. T. D. Colmer, T. J. Flowers, R. Munns, Use of wild relatives to improve salt tolerance in wheat. J. Exp. Bot. 57, 1059-1078 (2006).

31. R. S. Pinto, G. Molero, M. P. Reynolds, Identification of heat tolerant wheat lines showing genetic variation in leaf respiration and other physiological traits. Euphytica 213 (2017).

32. R. S. Pinto, et al., Heat and drought adaptive QTL in a wheat population designed to minimize confounding agronomic effects. Theor. Appl. Genet. 121, 1001-1021 (2010).

33. R. S. Pinto, M. P. Reynolds, Common genetic basis for canopy temperature depression under heat and drought stress associated with optimized root distribution in bread wheat. Theor. Appl. Genet. 128, 575-585 (2015).

34. I. Amani, R. A. Fischer, M. P. Reynolds, Evaluation of canopy temperature as a screening tool for heat tolerance in spring wheat. J. Agron. Crop Sci. 176, 119-129 (1996).

35. Q. Song, et al., Optimal crop canopy architecture to maximise canopy photosynthetic CO2 uptake under elevated $\mathrm{CO} 2$ - a theoretical study using a mechanistic model of canopy photosynthesis.

Funct. Plant Biol. 40, 108-124 (2013). 
36. M. Reynolds, et al., Breeder friendly phenotyping. Plant Sci. 295, 110396 (2020).

37. L.-J. Gardiner, R. Joynson, A. Hall, Next-Generation Sequencing Enabled Genetics in Hexaploid Wheat. Appl. Genet. Genomic Res. Cereal., 49-63 (2019).

38. M. Hao, et al., The Resurgence of Introgression Breeding, as Exemplified in Wheat Improvement. Front. Plant Sci. 11, 252 (2020).

39. K. Gaurav, et al., Population genomic analysis of Aegilops tauschii identifies targets for bread wheat improvement. Nat. Biotechnol. 2021, 1-10 (2021).

40. R. D. Argyros, et al., Type B Response Regulators of Arabidopsis Play Key Roles in Cytokinin Signaling and Plant Development. Plant Cell 20, 2102 (2008).

41. M. Frank, A. Cortleven, O. Novák, T. Schmülling, Root-derived trans-zeatin cytokinin protects Arabidopsis plants against photoperiod stress. Plant. Cell Environ. 43, 2637-2649 (2020).

42. K. H. Nguyen, et al., Arabidopsis type B cytokinin response regulators ARR1, ARR10, and ARR12 negatively regulate plant responses to drought. Proc. Natl. Acad. Sci. U. S. A. 113, 3090-3095 (2016).

43. X. Huang, et al., The Antagonistic Action of Abscisic Acid and Cytokinin Signaling Mediates Drought Stress Response in Arabidopsis. Mol. Plant 11, 970-982 (2018).

44. Y. Liu, M. Zhang, Z. Meng, B. Wang, M. Chen, Research Progress on the Roles of Cytokinin in Plant Response to Stress. Int. J. Mol. Sci. 21, 6574 (2020).

45. G. Ning, et al., Genetic manipulation of Soc1-like genes promotes photosynthesis in flowers and leaves and enhances plant tolerance to high temperature. Plant Biotechnol. J. 19, 8-10 (2021).

46. B. Coombes, et al., Whole genome sequencing uncovers the structural and transcriptomic landscape of hexaploid wheat/Am. muticum introgression lines. bioRxiv, 2021.11.16.468825 (2021).

47. R. R. Kumar, et al., Characterizing the putative mitogen-activated protein kinase (MAPK) and their protective role in oxidative stress tolerance and carbon assimilation in wheat under terminal heat stress. Biotechnol. Reports 29, e00597 (2021).

48. S. Mo, et al., Mitogen-activated protein kinase action in plant response to high-temperature stress: a mini review. Protoplasma 258, 477-482 (2021). 
49. Y. Zhou, et al., Overexpression of soybean DREB1 enhances drought stress tolerance of transgenic wheat in the field. J. Exp. Bot. 71, 1842-1857 (2020).

50. S. ji Lee, et al., DREB2C Interacts with ABF2, a bZIP Protein Regulating Abscisic AcidResponsive Gene Expression, and Its Overexpression Affects Abscisic Acid Sensitivity. Plant Physiol. 153, 716-727 (2010).

51. M. K. Mishra, et al., Overexpression of WsSGTL1 Gene of Withania somnifera Enhances Salt Tolerance, Heat Tolerance and Cold Acclimation Ability in Transgenic Arabidopsis Plants. PLoS One 8 (2013).

52. P. Misra, G. Singh, M. K. Mishra, V. Pandey, S. Saema, Functional Analysis and the Role of Members of SGT Gene Family of Withania somnifera. Transgenes. Second. Metab., 1-14 (2016).

53. W. Xiong, et al., Increased ranking change in wheat breeding under climate change. Nat. Plants 202179 7, 1207-1212 (2021).

54. K. D. Sayre, S. Rajaram, R. A. Fischer, Yield Potential Progress in Short Bread Wheats in Northwest Mexico. Crop Sci. 37, 36-42 (1997).

55. J. C. ZADOKS, T. T. CHANG, C. F. KONZAK, A decimal code for the growth stages of cereals. Weed Res. 14, 415-421 (1974).

56. A. Pask, J. Pietragalla, D. Mullan, M. P. Reynolds, Physiological breeding II: a field guide to wheat phenotyping. CIMMYT (2012) (January 18, 2022).

57. R. A. Fischer, R. Maurer, Drought resistance in spring wheat cultivars. I. Grain yield responses. Aust. J. Agric. Res. 29, 897-912 (1978).

58. G. Alvarado, et al., META-R (Multi Environment Trail Analysis with R for Windows) Version 6.04 https:/doi.org/hdl:11529/10201.

59. H. Li, et al., The Sequence Alignment/Map format and SAMtools. Bioinformatics 25, 2078 (2009).

60. H. Li, A statistical framework for SNP calling, mutation discovery, association mapping and population genetical parameter estimation from sequencing data. Bioinformatics 27, 2987 (2011).

61. P. Cingolani, et al., A program for annotating and predicting the effects of single nucleotide polymorphisms, SnpEff: SNPs in the genome of Drosophila melanogaster strain w1118; iso-2; iso-3. Fly (Austin). 6, 80 (2012). 
62. A. E. Lipka, et al., GAPIT: genome association and prediction integrated tool. Bioinformatics 28, 2397-2399 (2012).

63. J. K. Pritchard, M. Stephens, P. Donnelly, Inference of Population Structure Using Multilocus Genotype Data. Genetics 155, 945-959 (2000).

64. M. K. Hyun, et al., Efficient Control of Population Structure in Model Organism Association Mapping. Genetics 178, 1709-1723 (2008).

65. A. R. Quinlan, I. M. Hall, BEDTools: a flexible suite of utilities for comparing genomic features. Bioinformatics 26, 841-842 (2010).

66. B. S. Pedersen, A. R. Quinlan, hts-nim: scripting high-performance genomic analyses. Bioinformatics 34, 3387 (2018).

67. J. Keilwagen, et al., Detecting Large Chromosomal Modifications Using Short Read Data From Genotyping-by-Sequencing. Front. Plant Sci. 10, 1133 (2019).

68. E. Bauer, et al., Towards a whole-genome sequence for rye (Secale cereale L.). Plant J. 89, 853869 (2017).

69. S. Walkowiak, et al., Multiple wheat genomes reveal global variation in modern breeding. Nat. 20205887837 588, 277-283 (2020).

70. H. Li, Minimap2: pairwise alignment for nucleotide sequences. Bioinformatics 34, 3094 (2018).

71. D. Winter, pafr: Read, Manipulate and Visualize "Pairwise mApping Format" Data (2021).

72. C. Camacho, et al., BLAST+: Architecture and applications. BMC Bioinformatics 10, 1-9 (2009).

73. S. C. Potter, et al., HMMER web server: 2018 update. Nucleic Acids Res. 46, W200 (2018).

74. D. Kim, J. M. Paggi, C. Park, C. Bennett, S. L. Salzberg, Graph-based genome alignment and genotyping with HISAT2 and HISAT-genotype. Nat. Biotechnol. 2019378 37, 907-915 (2019).

75. C. Trapnell, et al., Differential gene and transcript expression analysis of RNA-seq experiments with TopHat and Cufflinks. Nat. Protoc. 201273 7, 562-578 (2012).

76. J. T. Robinson, et al., Integrative genomics viewer. Nat. Biotechnol. 2011291 29, 24-26 (2011).

77. P. Rice, L. Longden, A. Bleasby, EMBOSS: the European Molecular Biology Open Software Suite. Trends Genet. 16, 276-277 (2000). 
bioRxiv preprint doi: https://doi.org/10.1101/2022.02.09.479695; this version posted February 10,2022 . The copyright holder for this preprint

(which was not certified by peer review) is the author/funder, who has granted bioRxiv a license to display the preprint in perpetuity. It is made available under aCC-BY-ND 4.0 International license.

78. M. Pertea, et al., StringTie enables improved reconstruction of a transcriptome from RNA-seq reads. Nat. Biotechnol. 2015333 33, 290-295 (2015).

79. P. Borrill, R. Ramirez-Gonzalez, C. Uauy, expVIP: a Customizable RNA-seq Data Analysis and Visualization Platform. Plant Physiol. 170, 2172-2186 (2016). 